\title{
A Quantity Model for Controlling and Measuring Software Quality Based on the Expert Decision-Making Algorithm
}

\author{
Che-Wei CHANG ${ }^{1}$, Der-Juinn HORNG ${ }^{2}$, Hung-Lung LIN ${ }^{2}$ \\ ${ }^{I}$ Department of Information Management, Yuanpei University, Hsin Chu, Taiwan, China \\ ${ }^{2}$ Department of Business Administration, National Central University, Jhongli City, Taiwan, China \\ Email:chewei@mail.ypu.edu.tw,horng@cc.ncu.edu.tw,hsa8936.hsa8936@msa.hinet.net
}

\begin{abstract}
Researchers have been active in the field of software engineering measurement over more than 30 years. The software quality product is becoming increasingly important in the computerized society. Target setting in software quality function and usability deployment are essential since they are directly related to development of high quality products with high customer satisfaction. Software quality can be measured as the degree to which a particular software program complies with consumer demand regarding function and characteristics. Target setting is usually subjective in practice, which is unscientific. Therefore, this study proposes a quantity model for controlling and measuring software quality via the expert decision-making algorithm-based method for constructing an evaluation method can provide software in relation to users and purchasers, thus enabling administrators or decision makers to identify the most appropriate software quality. Importantly, the proposed model can provide s users and purchasers a reference material, making it highly applicable for academic and government purposes.
\end{abstract}

Keywords: software quality characteristics, software quality model, multiple criteria decision making (MCDM), analytic hierarchy process (AHP)

\section{Introduction}

The numerous challenges involved in software development include devising quality software, accurately controlling overhead costs, complying with a progress schedule, maintaining the software system, coping with unstable software systems and satisfying consumer demand with respect to software quality [1-3]. These challenges may incur a software development crisis if performed inefficiently. Problems within the software sector can be summed up as follows: 1) Inability to accurately forecast or control software development costs, 2) substandard quality, poor reliability and ambiguous requests on how to enhance requests by management directives regarding, 3) unnecessary risks while offering and maintaining quality assurance, and 4) high personnel turnover rate, leading to lack of continuity and increased incidence of defects in software development [4].

Researchers have been active in the field of software engineering measurement over more than 30 years. The software quality product is becoming increasingly important in the computerized society. Developing software quality is complex and difficult, and so a firm must maintain the software quality to gain a competitive advantage. However, when software quality is being de- veloped, developers must simultaneously consider developmental budget, the schedule, the ease of maintenance of the system and the user's requirements. Users' requirements and the management of software firms together determine the implementation of software systems.

Target setting in software quality function and usability deployment are essential since they are directly related to development of high quality products with high customer satisfaction. Software quality can be measured as the degree to which a particular software program complies with consumer demand regarding function and characteristics (degree of conformity to requirements). However, target setting is usually subjective in practice, which is unscientific $[5,6]$. Therefore, this study proposes a quantity model for controlling and measuring software quality via the expert decision-making algorithm-based method for constructing an evaluation method can provide software in relation to users and purchasers, thus enabling administrators or decision makers to identify the most appropriate software quality. Importantly, the proposed model can provide $\mathrm{s}$ users and purchasers a reference material, making it highly applicable for academic and government purposes. 


\section{Model for the Software Quality}

Conversely, capability assessment frameworks usually assess the process that is followed on a project in practice in the context of a process reference model, defined separately and independently of any particular methodology [7]. Software architecture is a key asset in any organization that builds complex software-intensive systems. Because of its central role as a project blueprint, organizations should first analyze the architecture before committing resources to a software development program [8]. To resolve the above problems, multi-attribute characteristics or factors of software quality must be considered. Effective management strategies in a technology setting are thus essential for resolving crises in software development. Technological aspects are software technology adopted and design expertise of a software developer [4].

Multiple criteria decision making (MCDM) is a methodology that helps decision makers make preference decisions (e.g. assessment, ranking, selection) regarding a finite set of available alternatives (courses of action) characterized by multiple, potentially conflicting attributes $[9,10]$. MCDM provides a formal framework for modeling multi-attribute decision problems, particularly problems whose nature demands systematic analysis, including analysis of decision complexity, regularity, significant consequences, and the need for accountability [9]. Among those well-known methods, MCDM has only relatively recently been employed to evaluate software quality performance. MCDM-based decision-making is a wide method for the measuring the software quality [4,11-13]. Among those well-known evaluation methods, MCDM has been employed relatively recently to evaluate organizational performance and it uses a set of attributes to resolve decision-making issues. Currently, one of the most popular existing evaluation techniques was performed by adopting the analytic hierarchy process (AHP), which was utilized by setting up hierarchical or skeleton within which multi-attribute decision problems can be structured [13-18].

\subsection{Analytic Hierarchic Process (AHP)}

Assume that we have $\mathrm{n}$ different and independent criteria $\left(C_{1}, C_{2}, \ldots, C_{n}\right)$ and they have the weights $\left(W_{1}, W_{2}, \ldots\right.$, $\left.W_{n}\right)$, respectively. The decision-maker does not know in advance the values of $W_{\mathrm{i}}, i=1,2, \ldots, n$, but he is capable of making pair-wise comparison between the different criteria. Also, assume that the quantified judgments provided by the decision-maker on pairs of criteria $\left(C_{i}, C_{j}\right)$ are represented in an $n \times n$ matrix as in the following:

$$
\begin{aligned}
& \begin{array}{llll}
C_{1} & C_{2} & \cdots & C_{n}
\end{array}
\end{aligned}
$$

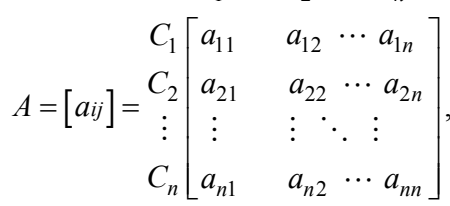

If for example the decision-maker compares $C_{1}$ with $C_{2}$, he provides a numerical value judgment $a_{12}$ which should represent the importance intensity of $C_{1}$ over $C_{2}$. The $a_{12}$ value is supposed to be an approximation of the relative importance of $C_{1}$ to $C_{2}$; i.e., $a_{12} \approx\left(W_{1} / W_{2}\right)$. This can be generalized and the following can be concluded:

1) $a_{12} \approx\left(W_{1} / W_{2}\right) i, j=1,2, \ldots, n$.

2) $a_{i i}=1, i=1,2, \ldots, n$.

3) If $a_{i j}=\delta, \delta \neq 0$, than $a_{j i}=1 / \delta, i=1,2, \ldots, n$.

4) If $C_{i}$ is more important than $C_{j}$, than $a_{12} \approx$ $\left(W_{1} / W_{2}\right)>1$.

This implies that matrix $A$ should be a positive and reciprocal matrix with 1's in the main diagonal and hence the decision-maker needs only to provide value judgments in the upper triangle of the matrix. The values assigned to $a_{i j}$ according to Saaty scale are usually in the interval of $1-9$ or their reciprocals. Table 1 presents Saaty's scale of preferences in the pair-wise comparison process. It can be shown that the number of judgments $(L)$ needed in the upper triangle of the matrix are:

$$
L=n(n-1) / 2,
$$

where $n$ is the size of the matrix $A$ :

Having recorded the numerical judgments aij in the matrix $A$, the problem now is to recover the numerical weights $\left(W_{1}, W_{2}, \ldots, W_{n}\right)$ of the criteria from this matrix. In order to do so, consider the following equation:

$$
\begin{gathered}
{\left[\begin{array}{cccc}
\tilde{a}_{11} & \tilde{a}_{12} & \cdots & \tilde{a}_{1 n} \\
\tilde{a}_{21} & \tilde{a}_{22} & \cdots & \tilde{a}_{2 n} \\
\vdots & \vdots & \ddots & \vdots \\
\tilde{a}_{n 1} & \tilde{a}_{n 2} & \cdots & \tilde{a}_{n n}
\end{array}\right]} \\
\approx\left[\begin{array}{cccc}
W_{1} / W_{1} & W_{1} / W_{2} & \cdots & W_{1} / W_{n} \\
W_{2} / W_{1} & W_{2} / W_{2} & \cdots & W_{2} / W_{n} \\
\vdots & \vdots & \ddots & \vdots \\
W_{n} / W_{1} & W_{n} / W_{2} & \cdots & W_{n} / W_{n}
\end{array}\right] .
\end{gathered}
$$

Moreover, by multiplying both matrices in Equation (3) on the right with the weights vector $W=\left(W_{1}, W_{2}, \ldots, W_{n}\right)$, where $W$ is a column vector. The result of the multiplication of the matrix of pair-wise ratios with $W$ is $n W$, hence it follows:

$$
A \times W=n \times W .
$$

This is a system of homogenous linear equations. It has a non-trivial solution if and only if the determinant of $A-n I$ vanishes, that is, $n$ is an eigenvalue of $A . I$ is an $n \times n$ identity matrix. Saaty's method computes $W$ as the principal right eigenvector of the matrix $A$, that is, 
Table 1. Saaty's scale of preferences in the pair-wise comparison process

\begin{tabular}{cl}
\hline Numerical & \multicolumn{1}{c}{ Verbal judgments of preferences between $C_{i}$ and $C_{j}$} \\
\hline 1 & $i$ is equally important to $j$ \\
5 & $i$ is slightly more important than $j$ \\
7 & $i$ is strongly more important than $j$ \\
9 & $i$ is very strongly more important than $j$ \\
$2,4,6,8$ & $i$ is extremely more important than $j$ \\
\hline
\end{tabular}

Table 2. Average random index for corresponding matrix size

\begin{tabular}{cccccccccccccccc}
\hline$(n)$ & 1 & 2 & 3 & 4 & 5 & 6 & 7 & 8 & 9 & 10 & 11 & 12 & 13 & 14 & 15 \\
\hline$($ R.I. $)$ & 0 & 0 & 0.58 & 0.90 & 1.12 & 1.24 & 1.32 & 1.41 & 1.45 & 1.49 & 1.51 & 1.48 & 1.56 & 1.57 & 1.59 \\
\hline
\end{tabular}

$$
A \times W=\lambda_{\max } W
$$

where $\lambda_{\max }$ is the principal eigenvalue of the matrix $A$.

If matrix $A$ is a positive reciprocal one then $\lambda_{\max } \geq n$, [19]. The judgments of the decision-maker are perfectly consistent as long as

$$
a_{i j} a_{j k}=a_{i k}, i, j, k=1,2, \ldots, n,
$$

which is equivalent to

$$
\left(W_{i} / W_{j}\right)\left(W_{j} / W_{k}\right)=\left(W_{i} / W_{k}\right),
$$

The eigenvector method yields a natural measure of consistency. Saaty defined the consistency index (C.I.) as

$$
\text { C.I. }=\left(\lambda_{\max }-n\right) /(n-1) \text {. }
$$

For each size of matrix $n$; random matrices were generated and their mean C.I. value, called the random index (R.I.), was computed and tabulated as shown in Table 2. Accordingly, Saaty defined the consistency ratio as

$$
\text { C.R. }=\text { C.I / R.I. . }
$$

The consistency ratio C.R. is a measure of how a given matrix compares to a purely random matrix in terms of their consistency indices. A value of the consistency ratio C.R. $\leq 0.1$ is considered acceptable. Larger values of C.R. require the decision-maker to revise his judgments.

\section{Case Implementation}

The city government of Hsinchu, in northern Taiwan, intends to implement a system for monitoring public spaces. Hence, the Hsinchu City Government is installing digital video recorder systems (DVRs). in place of traditional surveillance camera systems. The DVRs circumvents restrictions corrects the limitations of traditional surveillance camera systems, which include: a) lapses in recording due to operator neglect or machine error; b) difficulty in locating a desired time sequence following completion of a recording; c) poor video quality and d) difficulty in maintaining and preserving tapes due to a lack storage space and natural degradation of film quality.

According to government procurement regulations, regional governments must select at least five evaluators to review more than three firms before evaluating the best DVRs software quality. This study considers four candidate DVRs software packages common in the surveillance market manufactured by Firms A, B, C and D.

As Figure 1 shows, the ISO 9126-1 standard was developed in 2001 not only to identify major quality attributes of computer software, but also as a measure of six major quality attributes. The proposed method adopts the ISO 9126-1 model to evaluate the DVRs software quality. The applicability of the proposed model is demonstrated in a case study. This model for evaluating the DVRs software quality comprises the following steps.

\subsection{Step 1: Establish an Evaluation Model and Define the Criteria}

Evaluate the ideal model, as six evaluation criteria, twenty-one sub-criteria and, finally, comparison with four alternatives (Figure 1). The evaluation criteria and sub-criteria used to evaluate the DVRs software quality are defined as follows:

- Functionality $\left(\mathrm{C}_{1}\right)$ : The degree to which the software satisfies stated requirements, including the four sub-criteria of suitability, accuracy, interoperability and security.

- Suitability: capability of software to provide an appropriate set of functions for specified tasks and user objectives.

- Maturity: capability of software to avert failure caused by software defects. 


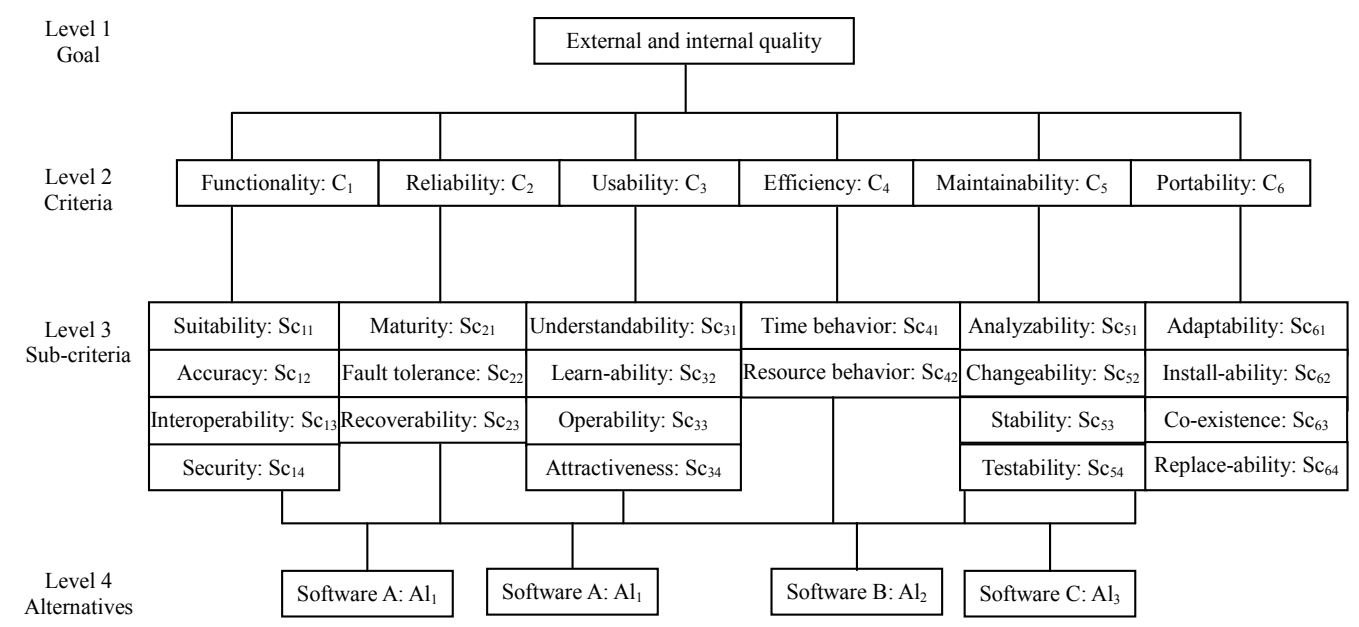

Figure 1. The ISO 9126-1 evaluate model

- Fault tolerance: capability of software to maintain a specified performance level in case of software errors or infringement of its specified interface.

- Accuracy: capability of software to provide correct or anticipated results or effects.

- Interoperability: capability of software to interact with one or more specified systems.

- Security: capability of software to prevent prohibited access and withstand deliberate attacks intended to gain unauthorized access to confidential information, or to make unauthorized access.

- Reliability $\left(\mathrm{C}_{2}\right)$ : How long the software is available for use; this includes the three sub-criteria of maturity, fault tolerance, and recoverability.

- Recoverability: capability of software to re-establish its level of performance and recover the data directly affected if a failure occurs.

- Usability $\left(\mathrm{C}_{3}\right)$ : Ease of implementation, including the four sub-criteria of understandability, learn-ability, operability and attractiveness.

- Understandability: capability of software to enable users to understand the appropriateness of a software and its use for particular tasks and conditions of use.

- Learning ability: capability of software to enable users to learn its application.

- Operability: capability of software to enable users to operate and control it.

- Attractiveness: capability of software to gain user acceptance.

- Efficiency $\left(\mathrm{C}_{4}\right)$ : Optimal use of system resources, including the two sub-criteria of time behavior and resource behavior.

- Time behavior: capability of software to provide appropriate responses, processing times and throughput rates when performing its function under stated conditions.

- Resource behavior: capability of software to use appropriate resources in time when the software implements its function under stated conditions.

- Maintainability $\left(\mathrm{C}_{5}\right)$ : The ease of which repairs can be made to the software, including the four sub-criteria of analyzability, changeability, stability and testability.

- Analyzability: capability of software to be diagnosed for deficiencies or causes of failures in the software or for identification of parts requiring modification.

- Changeability: capability of software to enable a specified modification to be implemented.

- Stability: ability of software to minimize unexpected effects from software modifications.

- Testability: ability of software to validate modified software.

- Portability $\left(\mathrm{C}_{6}\right)$ : How easily the software can be transposed from one environment to another; including four sub-criteria of adaptability install ability, co existence and replace ability.

- Adaptability: capability of software to be modified for specified environments without applying actions or means other than those provided for the software considered.

- Install-ability: capability of software to be installed in a specified environment.

- Co-existence: capability of software to co-exist with other independent software in a common environment sharing common resources.

- Replace-ability: capability of software to replace other specified software in the environment of that software.

\subsection{Step 2: Establish the Pair-Wise Comparison Matrix and Determine Consistency}

Twenty one experts are assigned and rated on a ninepoint scale against each criterion to assess criteria and sub-criteria. The experts were proficient in PC modules, 
software modules and network communication modules. The program adopted of the respondents' data to crosscompare all criteria and alternatives to determine the weights and inconsistency ratios. The inconsistency ratio is a measure of the percentage of time when decision makers are inconsistent in making judgment. The "acceptable" inconsistency ratio was approximately 0.1 or less, but "particular circumstance" may warrant the acceptance of a higher value. However, an inconsistency ratio of 1 is unacceptable because the ratings are as good as random judgments. Four of the twenty one experts had inconsistency ratios above 0.15 . This was too high and their responses were discarded. Of the remaining seventeen, ten experts had low inconsistency ratios $(<0.05)$, and seven had ratios between 0.12 and 0.14 . These seven respondents were each given another chance to recheck at their ratings and determine whether they would like to modify their decisions, and eventually modified their rating by making their own adjustments to the data. Chang et al. proposal determining consistency procedure as shows Figure 2.

After discarding ht responses of the four inconsistent experts, the weights were then determined for a sample group of seventeen individuals matching the above characteristics with each respondent, making a pair-wise comparison of the decision elements and assigning them relative scores.

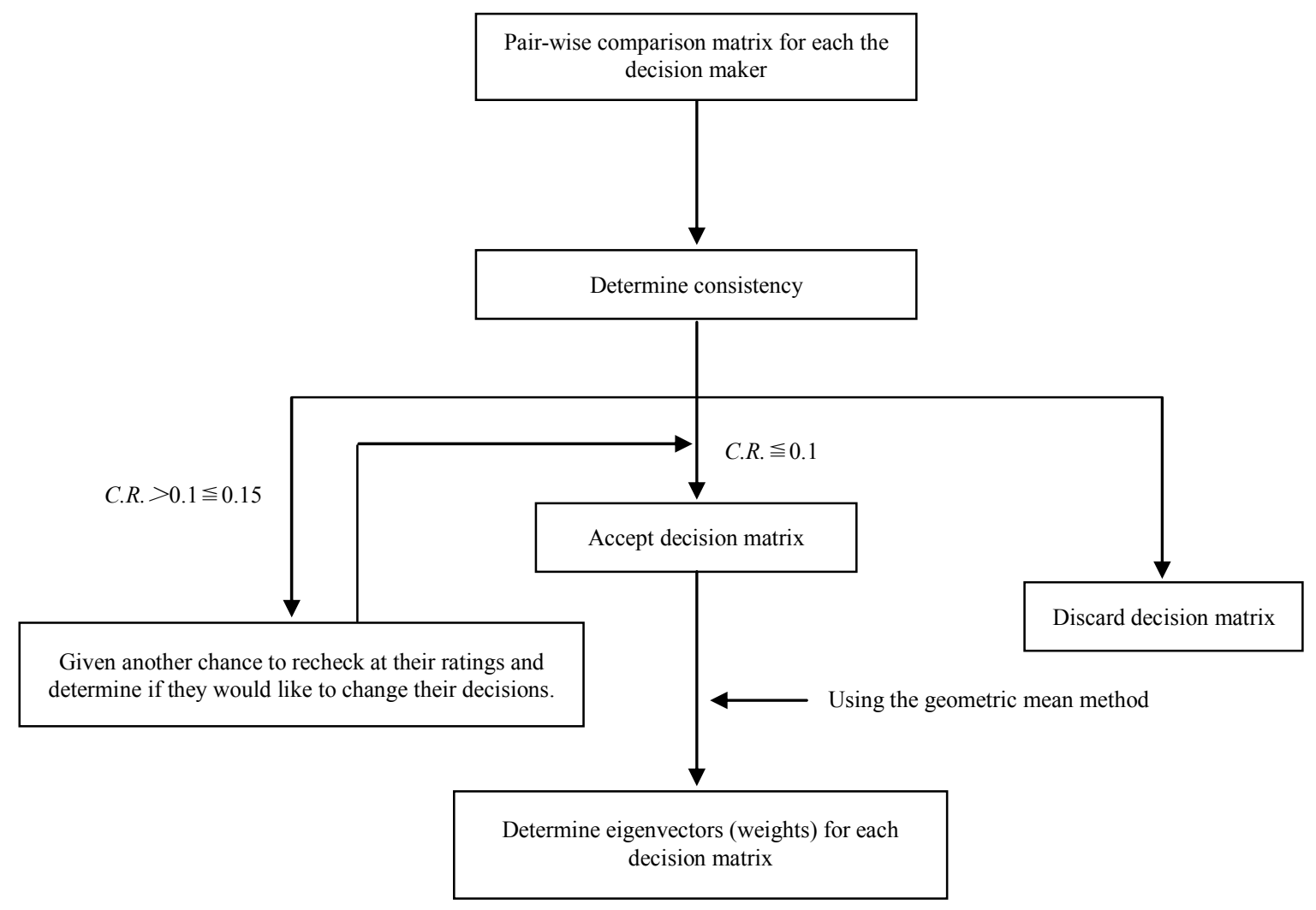

Figure 2. The procedure for determining consistency

Table 3. Aggregate pair-wise comparison matrix with eigenvectors

\begin{tabular}{cccccccc}
\hline & $C_{1}$ & $C_{2}$ & $C_{3}$ & $C_{4}$ & $C_{5}$ & $C_{6}$ & Eigenvectors \\
\hline$C_{1}$ & 1.000 & 0.812 & 0.474 & 0.321 & 0.722 & 1.182 & 0.108 \\
$C_{2}$ & 1.231 & 1.000 & 0.762 & 0.695 & 0.433 & 2.150 & 0.147 \\
$C_{3}$ & 2.110 & 1.313 & 1.000 & 0.913 & 1.167 & 1.909 & 0.207 \\
$C_{4}$ & 3.120 & 1.438 & 1.095 & 1.000 & 1.278 & 2.110 & 0.241 \\
$C_{5}$ & 1.385 & 2.310 & 0.857 & 0.782 & 1.000 & 1.636 & 0.198 \\
$C_{6}$ & 0.846 & 0.465 & 0.524 & 0.474 & 0.611 & 1.000 & 0.098 \\
\hline \multicolumn{7}{c}{$\lambda_{\max }=6.118$, C.I. $=0.024$, R I. $=1.24$, C.R. $=0.019$} \\
\hline
\end{tabular}


Table 4. Summarizes eigenvectors (weights) results for levels 2 to 4

\begin{tabular}{|c|c|c|c|c|c|c|c|c|}
\hline \multirow{2}{*}{ Criteria } & \multirow{2}{*}{$\begin{array}{l}\text { Weights } \\
\text { for level } 2\end{array}$} & \multirow{2}{*}{ Sub-Criteria } & \multirow{2}{*}{$\begin{array}{l}\text { Weights } \\
\text { for level } 3\end{array}$} & \multirow{2}{*}{$\begin{array}{l}\text { Weights of the } \\
\text { overall }\end{array}$} & \multicolumn{4}{|c|}{ Weights for level 4} \\
\hline & & & & & $A l_{1}$ & $\mathrm{Al}_{2}$ & $\mathrm{Al}_{3}$ & $\mathrm{Al}_{4}$ \\
\hline \multirow{4}{*}{$C_{1}$} & \multirow{4}{*}{0.108} & $S C_{1}$ & 0.207 & 0.041 & 0.290 & 0.300 & 0.152 & 0.258 \\
\hline & & $S C_{2}$ & 0.157 & 0.035 & 0.316 & 0.281 & 0.140 & 0.263 \\
\hline & & $S C_{3}$ & 0.258 & 0.052 & 0.278 & 0.260 & 0.180 & 0.282 \\
\hline & & $S C_{4}$ & 0.378 & 0.060 & 0.296 & 0.309 & 0.126 & 0.269 \\
\hline \multirow{3}{*}{$C_{2}$} & \multirow{3}{*}{0.147} & $S C_{5}$ & 0.236 & 0.067 & 0.266 & 0.465 & 0.105 & 0.164 \\
\hline & & $S C_{6}$ & 0.354 & 0.057 & 0.240 & 0.348 & 0.190 & 0.222 \\
\hline & & $S C_{7}$ & 0.410 & 0.037 & 0.343 & 0.280 & 0.162 & 0.216 \\
\hline \multirow{4}{*}{$C_{3}$} & \multirow{4}{*}{0.207} & $S C_{8}$ & 0.323 & 0.046 & 0.320 & 0.347 & 0.112 & 0.221 \\
\hline & & $S C_{9}$ & 0.275 & 0.114 & 0.344 & 0.221 & 0.112 & 0.323 \\
\hline & & $S C_{10}$ & 0.179 & 0.127 & 0.222 & 0.207 & 0.236 & 0.335 \\
\hline & & $S C_{11}$ & 0.223 & 0.058 & 0.217 & 0.140 & 0.372 & 0.271 \\
\hline \multirow{2}{*}{$C_{4}$} & \multirow{2}{*}{0.241} & $S C_{12}$ & 0.475 & 0.035 & 0.213 & 0.180 & 0.338 & 0.269 \\
\hline & & $S C_{13}$ & 0.525 & 0.043 & 0.329 & 0.289 & 0.111 & 0.271 \\
\hline \multirow{4}{*}{$C_{5}$} & \multirow{4}{*}{0.198} & $S C_{14}$ & 0.295 & 0.062 & 0.287 & 0.265 & 0.114 & 0.334 \\
\hline & & $S C_{15}$ & 0.177 & 0.017 & 0.289 & 0.330 & 0.126 & 0.254 \\
\hline & & $S C_{16}$ & 0.216 & 0.033 & 0.246 & 0.358 & 0.121 & 0.276 \\
\hline & & $S C_{17}$ & 0.312 & 0.024 & 0.330 & 0.306 & 0.100 & 0.264 \\
\hline \multirow{4}{*}{$C_{6}$} & \multirow{4}{*}{0.098} & $S C_{18}$ & 0.172 & 0.024 & 0.314 & 0.387 & 0.138 & 0.162 \\
\hline & & $S C_{19}$ & 0.336 & 0.041 & 0.406 & 0.246 & 0.103 & 0.245 \\
\hline & & $S C_{20}$ & 0.244 & 0.035 & 0.207 & 0.526 & 0.112 & 0.155 \\
\hline & & $S C_{21}$ & 0.248 & 0.052 & 0.271 & 0.478 & 0.089 & 0.161 \\
\hline
\end{tabular}

\subsection{Step 3: Determine Eigenvectors}

The relative scores provided by 11 experts were then aggregated by the geometric mean method. Table 3 presents the aggregate pair-wise comparison matrix and the consistency test for level 2. The eigenvectors (weighs) for level 2 can be determined by the procedure described in the previous section, and are as follows

$$
\left.\begin{array}{l|l}
C_{1} & 0.108 \\
C_{2} & 0.147 \\
C_{3} & 0.207 \\
C_{4} & 0.241 \\
C_{5} & 0.198 \\
C_{6} & 0.098
\end{array}\right] .
$$

The respective weights of the six evaluative criteria are functionality $(0.305)$, reliability $(0.255)$, usability $(0.160)$, efficiency (0.092), maintainability $(0.135)$ and portability $(0.053)$.
The eigenvectors (weighs) for level 3 can be determined by the procedure described in the previous section, and are as follows

The twenty-one evaluative sub-criteria are weighted as follows: suitability (0.207), accuracy (0.157), interoperability (0.258), security (0.378), maturity $(0.236)$, fault tolerance $(0.354)$, recoverability 0.410$)$, understandability (0.323), learn-ability $(0.275)$, operability $(0.179)$, attractiveness $(0.223)$, time behavior $(0.475)$, resource behavior (0.525), analyzability (0.295), changeability $(0.177)$, stability $(0.216)$, testability $(0.312)$, adaptability $(0.172)$, install-ability $(0.336)$, co-existence $(0.244)$ and replace-ability $(0.248)$. Table 4 summarizes eigenvectors (weights) results for levels 2 to 4 .

\subsection{Step 4: Determine DVRs' Software Quality}

According to Table 4, the quality of the four DVRs' software programs are then determined by Equation (12). Equation (12) indicates that the quality of the four DVRs' software programs are as follows: DVR A $=0.300, \mathrm{DVR}$ $\mathrm{B}=0.314$, DVR $\mathrm{C}=0.170$ and DVR $\mathrm{D}=0.277$. 
The DVR B performed the best; end users must test the stability of the system. The software products were tested on an Intel Pentium 4 3.2GB, 1GB DDR400 RAM $\mathrm{PC}$ and sixteen visual channels running Windows XP Professional. Therefore, the mean CPU efficiency was $32 \%$, and maximum efficiency was $43 \%$. The mean MEM loading was $10586 \mathrm{~K}$, and the top loading was 11200 K. During a week of testing, the system never crashed and never required automatic shutdown or restart. Clearly, DVR B had the best software quality.

\section{Conclusions}

This study proposes a multi-criteria evaluation model and algorithm capable of effectively evaluating software quality from the perspective of users or purchasers, thus enabling administrators or decision makers to identify optimum software quality. Significantly, this study provides procurement personnel with an easily applied and objective method of assessing the appropriateness of software quality. Therefore, this study proposes a method for identifying the best software quality from among those offered by four firms, by considering multiple assessment characteristics, improving upon the popular MCDM approach to alternative prioritization. This study presents an optimal operating model and algorithm for monitoring software in relation to users and purchasers, thus enabling administrators or decision makers to identify the most appropriate software quality. Based on the measurement results in this study, software users and developers can not only more thoroughly understand the merits and limitations of software products, but also ultimately enhance its overall quality. Administrators or decision makers adopt the measurement results of this study to evaluate software quality.

\section{REFERENCES}

[1] H. Aras, S. Erdogmus, and E. Koc, "Multi-criteria selection for a wind observation station location using analytic 
hierarchy process," Renewable Energy, Vol. 29, 2004, pp. 1383-1392.

[2] V. Belton and T. J. Stewart, "Multiple criteria decision analysis: An integrated approach," Kluwer Academic Publishers, Boston, 2002.

[3] G. P. Cesar, M. Tom, and H. S. Brian, "A Metamodel for assessable software development methodologies," Software Quality Journal, Vol. 13, No. 2, pp. 195-214, 2005.

[4] C. W. Chang, C. R. Wu, and H. L. Lin, "Evaluating the digital video recorder systems using analytic hierarchy and analytic network processes," Information Sciences, Vol. 177, No. 16, pp. 3383-3396, 2007.

[5] C. W. Chang, C. R. Wu, and H. L. Lin, (2007b), "Integrating fuzzy theory and hierarchy concepts to evaluate software quality," Software Quality Journal, Published online, Vol. 11, No. 27, 2007.

[6] C. W. Chang, C. R. Wu, and H. L. Lin, "Group decision-making in a multiple criteria environment-A case using the AHPGR model to assess digital video recorder systems," Journal of Testing and Evaluation, Vol. 36, No. 2, pp. 583-589, 2008.

[7] P. F. Hsu and B.-Y. Chen, "Developing and implementing a selection model for bedding chain retail store franchisee using Delphi and fuzzy AHP," Quality and Quantity, Vol. 41, No. 2, pp. 275-290, 2007.

[8] ISO/IEC9126-1, "Software engineering-product qualityPart1: Quality model," 2001.

[9] G. Issac, C. Rajendran, and R. N. Anantharaman, “An instrument for the measurement of customer perceptions of quality management in the software industry: An empirical study in India," Software Quality Journal, Vol. 14, No. 4, pp. 291-308, 2005.

[10] L. S. Jose and H. Ines, "An AHP-based methodology to rank critical success factors of executive information systems," Computer Standards and Interfaces, Vol. 28, pp. $1-12,2005$.

[11] R. Kazman, L. Bass, M. Klein, T. Lattanze, and L. Northrop, "A Basis for Analyzing Software Architecture
Analysis Methods," Software Quality Journal, Vol. 13, No. 4, pp. 329-355, 2005.

[12] T. M. Khoshgoftaar, A. Herzberg, and N. Seliya, "Resource oriented selection of rule-based classification models: An empirical case study," Software Quality Journal, Vol. 14, No. 4, pp. 309-338, 2006.

[13] T. M. Khoshgoftaar, N. Seliya, and N. Sundaresh, "An empirical study of predicting software faults with casebased reasoning," Software Quality Journal, Vol. 14, No. 2, pp. 85-111, 2006.

[14] L. Z. Lin, and T. H. Hsu, "The qualitative and quantitative models for performance measurement systems: The agile service development," Quality \& Quantity, Vol. 42, No. 4, pp. 445-476, 2008.

[15] F. Liu, K. Noguchi, A. Dhungana, A. V. V. N. S. N. Srirangam, and P. Inuganti, "A quantitative approach for setting technical targets based on impact analysis in software quality function deployment," Software Quality Journal, Vol. 14, No. 2, pp. 113-134, 2005.

[16] M. Mollaghasemi and J. Pet-Edwards, "Making multipleobjective decisions," Los Alamitos, IEEE Computer Society Press, CA, 1997.

[17] T. Rafla, P. N. Robillard, and M. C. Desmarais, (2007), "A method to elicit architecturally sensitive usability requirements: Its integration into a software development process," Software Quality Journal, Vol. 15, No. 2, pp. $117-133$.

[18] T. L. Saaty, (1980), "The analytic hierarchy process," McGraw Hill, New York, NY.

[19] E. Tolgaa, M. L. Demircana, and C. Kahraman, “Operating system selection using fuzzy replacement analysis and analytic hierarchy process," International Journal of Production Economics, Vol. 97, pp. 89-117, 2005.

[20] C. R. Wu, C. W. Chang, and H. L. Lin, "FAHP sensitivity analysis for measurement nonprofit organizational performance," Quality \& Quantity, Vol. 42, No. 3, pp. 283-302, 2008. 